\title{
Evidências de Validade da Global Measure of Relationship Satisfaction (GMREL) em Três Amostras da População Portuguesa
}

\author{
Evidence for the Validity of the Global Measure of Relationship Satisfaction \\ (GMREL) in Three Portuguese Samples
}

\author{
Patricia Monteiro Pascoal ${ }^{*}$, Leonor B. Oliveira \& Catarina F. Raposo \\ Universidade de Lisboa, Lisboa, Portugal
}

\begin{abstract}
Resumo
A satisfação relacional representa um construto de pertinência reconhecida para a avaliação relacional e em estreita conexão com diversas valências da saúde. A Global Measure of Relationship Satisfaction (GMREL) é formada por cinco itens e pretende avaliar a satisfação global com o companheiro/a no contexto de uma relação íntima. Foram estudadas as evidências de validade de construto e precisão dos escores desta escala em três amostras da população portuguesa, nomeadamente uma amostra clínica de pessoas com problemas relacionais $(N=56)$, uma amostra comunitária de pessoas sem estes problemas $(N=95)$ e uma amostra da comunidade de ambos os géneros $(N=669)$. A Escala de Avaliação da Satisfação em Áreas da Vida Conjugal foi empregue para investigar a validade convergente do instrumento, a Body Appearance Cognitive Distraction Scale para a validade divergente e, por fim, o estudo da Roc Curve com a amostra clínica e da comunidade para a validade discriminante. Os resultados indicam que a escala apresenta boas qualidades psicométricas para utilização em contexto clínico e de investigação.

Palavras-chave: Satisfação relacional, adaptação de instrumentos, validade de construto.
\end{abstract}

\begin{abstract}
Relationship satisfaction represents a well-known, pertinent construct for relationship assessment with strong connection to several health ranges. The Global Measure of Relationship Satisfaction (GMREL) is composed of five items and aims to evaluate the global satisfaction with the partner in an intimate relationship context. The purpose of this study was to observe the psychometric qualities of this scale in three Portuguese samples, namely a clinical sample of individuals with marital problems $(N=56)$, a normative sample of individuals without these problems $(N=95)$ and a community sample of both genders $(N=669)$. The Marital Life Areas Satisfaction Evaluation Scale was used to investigate the instrument's convergent validity; the Body Appearance Cognitive Distraction Scale for the divergent validity, and the Roc Curve study of clinical and community samples was used to assess the discriminant validity. The results show that the instrument presents good psychometric qualities for clinical and investigation purposes.

Keywords: Relationship satisfaction, instrument adaptation, construct validity.
\end{abstract}

A satisfação relacional representa um construto central à compreensão do funcionamento do casamento e das relações em geral, sendo o seu estudo da maior pertinência para a investigação e tratamento (Funk \& Rogge, 2007). A satisfação relacional tem sido associada à saúde mental (Wade \& Pevalin, 2004), especificamente em estudos da depressão, ansiedade ou uso de substâncias (Fincham \& Beach, 2010) e à saúde em geral, nomeadamente em

\footnotetext{
" Endereço para correspondência: Consulta de Sexologia Clinica, Faculdade de Psicologia, Universidade de Lisboa, Alameda da Universidade, Lisboa, Portugal 1649-013. E-mail: pmpascoal@psicologia.ulisboa.pt
}

estudos relacionados com o sistema imunitário, coração, diabetes ou cancro (Whisman, 2007).

Alguns benefícios para a sociedade, como a desistência do crime (Laub, Nagin, \& Sampson, 1998) têm também sido implicados com a satisfação relacional. Tem ainda sido consistentemente demonstrada a associação positiva entre satisfação relacional e satisfação sexual (e.g., Lawrance \& Byers, 1995; McGuire \& Barber, 2010) que, por sua vez, é um constructo central para a saúde sexual. Segundo a Organização Mundial de Saúde (OMS) o conceito de saúde sexual é um estado de bem-estar físico, emocional, mental e social relativamente à sexualidade e não somente a ausência de doença, disfunção ou enfermidade. A saúde sexual implica assim uma abordagem positiva e 
respeitadora da sexualidade e dos relacionamentos sexuais, bem como a possibilidade de experienciar contactos sexuais prazerosos e seguros, livres de coerção, discriminação e violência (World Health Organization, 2006).

De acordo com Vaughn e Baier (1999), a pertinência da satisfação relacional para a avaliação relacional encontra-se plenamente reconhecida, apesar de ter vindo a ser estudada sob diversas, e nem sempre sobrepostas formas, nomeadamente "satisfação matrimonial", "estabilidade", "qualidade", "ajustamento" ou "felicidade". A satisfação relacional resulta, antes, da apreciação pessoal e subjetiva da qualidade da relação (e.g., Narciso \& Ribeiro, 2009; Vaughn \& Baier, 1999). Tal concetualização inclui-se no Interpersonal Exchange Model of Sexual Satisfaction (IEMSS; Lawrance \& Byers, 1995), modelo a partir do qual foi desenvolvida a GMREL, que defende que a avaliação global das relações humanas se baseia na apreciação dos aspetos negativos (custos) em contraposição aos positivos (recompensas), tendo por base a Teoria das Trocas Sociais de Thibaut e Kelly (1959, citados por Vaughn \& Baier, 1999). Na revisão de literatura de Bradbury, Fincham e Beach (2000), os autores apontam ainda que a satisfação relacional consiste numa atitude para com o companheiro, que pode variar no grau e força que flutua no tempo. O construto tem sido predominantemente investigado no contexto hétero-normativo (Cusack, Hughes, \& Cook, 2012), tendo sofrido a diferenciação entre satisfação relacional global e satisfação específica com diferentes áreas da vida conjugal (Narciso \& Ribeiro, 2009). Pode ainda acrescentar-se que, uma vez que a satisfação relacional tem sido sobretudo estudada no contexto da conjugalidade, diversas outras estruturas relacionais permanecem ainda pouco examinadas, sendo conveniente um instrumento versátil.

As maiores críticas apontadas às escalas de satisfação relacional remetem para a sua extensão e resultante desadequação para fins clínicos ou de investigação (Hendrick, 1988) e para a falta de estudos de validação ou evidências de validade e fiabilidade (Funk \& Rogge, 2007; Vaughn \& Baier, 1999). Nos estudos de Funk e Rogge (2007) foram avaliadas a validade de construto e fiabilidade de várias escalas de satisfação relacional. Os autores concluíram que estas não são muito informativas devido à grande heterogeneidade de itens que contribuem sobretudo para a variância de erro.

Relativamente aos instrumentos de medição da satisfação relacional, estes são essencialmente escalas de autoavaliação (Funk \& Rogge, 2007) baseadas no vasto corpo de conhecimento que suporta a sua validade de construto (verificar a revisão de Bradbury et al., 2000). Muitos destes instrumentos são extensos e foram construídos para populações específicas. Salienta-se entre estas a Dyadic Adjustment Scale (DAS; Spanier, 1976), uma medida extensa para a qual já existem estudos de evidências de validade dos escores para a população portuguesa com 32 itens (Gomez \& Leal, 2008), sendo a medida mais citada de ajustamento relacional, originalmente desenhada para distinguir parceiros casados de divorciados (e.g., Christensen, Griffiths, \& Jorm, 2004), Na década de 90 foi criada a Global Measure of Relationship Satisfaction (GMREL; Lawrance \& Byers, 1995) cujo estudo para as evidências de validade e precisão nos propomos fazer no presente trabalho.

A nossa opção visa ter em conta as limitações existentes em diversas medidas tais como a extensão (Hendrick, 1988) e falta de estudos de validação, evidências de validade e de fiabilidade (Funk \& Rogge, 2007; Vaughn \& Baier, 1999) e, à semelhança de Bradbury et al., (2000) enfatizar o uso de medidas globais.

A GMREL consiste numa medida de satisfação relacional global desenvolvida a partir do Interpersonal Exchange Model of Sexual Satisfaction (IEMSS; Lawrance \& Byers, 1995), que tem demonstrado resultados estáveis e consistentes nos estudos psicométricos de validade de construto e de fiabilidade (Byers \& MacNeil, 2006) e foi já empregue em estudos com indivíduos em relações amorosas diversificadas (Byers, 2005; Byers \& Demmons, 1999; Byers, Demmons, \& Lawrance, 1998; MacNeil \& Byers, 2009; Peck, Shaffer, \& Williamson, 2004). A GMREL pode ser utilizada na generalidade das relações íntimas e em ambos os sexos, sendo um instrumento curto e de fácil compreensão além de uma medida específica da avaliação subjetiva da satisfação relacional. Tais vantagens motivaram a seleção deste instrumento para que se estudasse as evidências de validade para a população portuguesa.

$\mathrm{O}$ estudo da validade de construto e fiabilidade da GMREL foi efetuado por Lawrance e Byers (1995) numa população de homens e mulheres do Canadá, e posteriormente, por Byers e MacNeil (2006), também com homens e mulheres canadenses. Foi igualmente estudada numa amostra de casais heterossexuais chineses (Renaud, Byers, \& Pan, 1997), em amostras de indivíduos em relação de namoro (Byers \& Demmons, 1999; Byers et al., 1998; Peck et al., 2004) e em amostras de indivíduos em relações de longo termo (Byers, 2005; Byers \& Demmons, 1999; Byers et al., 1998; MacNeil \& Byers, 2009; Peck et al., 2004). A presente investigação sobre as evidências de validade psicométrica da GMREL junto da população portuguesa, comporta dois subestudos com três amostras diferentes: duas de recolha presencial (Subestudo 1); e outra de recolha online (Subestudo 2). Constituem, pois, os propósitos deste trabalho: (a) analisar a validade de construto (fatorial, convergente e divergente; Subestudos 1 e 2); (b) analisar a fiabilidade (consistência interna e correlação média interitens; Subestudos 1 e 2); (c) avaliar a sua capacidade discriminativa em relação aos participantes provenientes da amostra presencial clínica (com perceção de problemas relacionais), e da amostra presencial comunitária (sem perceção de problemas relacionais; Subestudo 1). 
Pascoal, P. M., Oliveira, L. B. \& Raposo, C. F. (2015). Evidências de Validade da Global Measure of Relationship Satisfaction (GMREL) em Três Amostras da População Portuguesa.

\section{Método}

\section{Participantes}

Subestudo 1. O Subestudo 1 foi conduzido presencialmente com duas amostras de conveniência da população portuguesa, comprometidos numa relação heterossexual de exclusividade em situação de coabitação. A amostra normativa é constituída por participantes que nas respostas ao protocolo de investigação aplicado, referiram não ter diagnóstico clínico conhecido e não revelaram autoperceção de problema relacional. Esta amostra inclui 95 participantes (22 homens e 72 mulheres), sendo a idade média de 29,3 $(D P=10,5)$, variando entre os 18 e os 57 anos (Tabela 1). Dada a prevalência de cerca de $90 \%$ de valores omissos, não foi calculada a média e desvio-padrão relativamente à duração da relação. A amostra clínica, com autoperceção e confirmação clínica de problemas relacionais, inclui 56 participantes (38 homens e 18 mulheres), tem uma idade média de 35,09 anos $(D P=11,29)$, variando entre os $21 \mathrm{e}$ os 71 anos. A duração média do casamento/união de facto é de 8,44 anos $(D P=8,1)$. A maioria dos participantes tem formação universitária (71\%).

Subestudo 2. Os 669 participantes (279 homens e 390 mulheres) da população portuguesa, com idade entre os 20 e os 65 anos e média de 36 anos $(D P=8,26)$, são provenientes de diferentes zonas do país. A duração do casamento/união de facto varia entre 1 e 39 anos e a média é de 7,5 anos $(D P=7,27)$.

Tabela 1

Caracterização Sócio- Demográfica das Amostras do Subestudo 1 e 2

\begin{tabular}{|c|c|c|c|}
\hline & \multicolumn{2}{|c|}{ Subestudo 1} & \multirow{2}{*}{$\begin{array}{c}\text { Subestudo } 2 \\
\text { Amostra Online } \\
N=669(\%)\end{array}$} \\
\hline & $\begin{array}{c}\text { Amostra Comunitária } \\
\qquad N=95(\%)\end{array}$ & $\begin{array}{c}\text { Amostra Clínica } \\
N=56(\%)\end{array}$ & \\
\hline \multicolumn{4}{|l|}{ Sexo } \\
\hline Masculino & $22(23,2)$ & $38(67)$ & $279(41,7)$ \\
\hline Feminino & $72(75,8)$ & $18(33)$ & $390(58,3)$ \\
\hline \multicolumn{4}{|l|}{ Hab. Literárias } \\
\hline até 4 anos & $3(3,2)$ & - & - \\
\hline $5^{\circ}-6^{\circ}$ ano & - & $3(5,4)$ & $1(0,1)$ \\
\hline $7^{\circ}-9^{\circ}$ ano & $2(2,1)$ & $6(10,7)$ & $20(3,0)$ \\
\hline $10^{\circ}-12^{\circ}$ & $7(7,4)$ & $7(12,5)$ & $68(10,2)$ \\
\hline Freq. Universitária & $46(48,4)$ & $10(17,9)$ & $70(10,7)$ \\
\hline Ensino Superior & $36(37,9)$ & $30(53,6)$ & $510(76,2)$ \\
\hline \multicolumn{4}{|l|}{ Zona de Residência } \\
\hline Norte & $27(28,4)$ & $4(3,9)$ & $83(12,4)$ \\
\hline Centro & $33(34,7)$ & $13(12,7)$ & $122(18,2)$ \\
\hline Grande Lisboa & $30(31,6)$ & $76(74,5)$ & $387(57,8)$ \\
\hline Alentejo & $1(1,1)$ & $1(1,0)$ & $19(2,8)$ \\
\hline Outra(s) & $2(2,1)$ & $6(5,9)$ & $58(8,6)$ \\
\hline
\end{tabular}

\section{Instrumentos (Subestudo 1 e Subestudo 2)}

Questionário Sociodemográfico. Foi elaborado um questionário para recolher dados sociodemográficos dos participantes, nomeadamente sexo, idade, habilitações literárias, estrutura relacional e orientação sexual (Tabela 1).

Global Measure of Relationship Satisfaction (GMREL; Lawrance \& Byers, 1995). Avalia a satisfação relacional global através da análise subjetiva que cada indivíduo efetua acerca da relação atual com o/a seu/sua companheiro/a. Os respondentes classificam a sua relação em cinco itens, numa escala de Likert de 7 pontos ("Muito Boa" - 7 a 1 - "Muito Má"; "Muito Agradável" - 7 a 1 - "Muito Desagradável"; "Muito Positiva" - 7 a 1 _ "Muito Negativa"; "Muito Satisfatória" - 7 a 1 - "Muito Insatisfatória"; "Muito Importante" - 7 a 1 - "Muito
Irrelevante"). As classificações são somadas permitindo valores entre 5 e 35 , sendo que resultados mais elevados indicam maior satisfação relacional.

Nos estudos iniciais, a GMREL apresentou boa consistência interna com um alfa de Cronbach de 0,90 (Lawrance \& Byers, 1995). A fiabilidade temporal no estudo original foi estudada com intervalos de aplicação de 2 semanas e 3 meses, e apresentou índice de correlação de $r=0,84$ entre as duas primeiras aplicações, e $r=0,78(p<0,001)$ entre a segunda e terceira aplicação (Lawrance \& Byers, 1995). Nos estudos originais verificou-se ainda a existência de adequada validade convergente com o Index of Sexual Satisfaction (Hudson, Harrison, \& Crosscup, 1981) com um índice de correlação de $r=0,70(p<0,001$; Byers et al., 1998; Lawrance \& Byers, 1995). 
Subescala da Sexualidade da Escala de Avaliação da Satisfação em Áreas da Vida Conjugal (EASAVIC; Narciso \& Costa, 1996). Para o estudo da validade convergente, foi empregue a Escala de Avaliação da Satisfação em Áreas da Vida Conjugal (EASAVIC), que consiste numa medida de autorrelato que avalia a satisfação conjugal global e em áreas específicas que compõem as subescalas de Intimidade, Funcionamento Familiar, Rede Social, Autonomia, Características Físicas, Família de Origem e Sexualidade. O instrumento compreende na sua globalidade 44 itens que avaliam a satisfação em diferentes áreas da vida conjugal numa escala de Likert de 6 pontos, desde "Nada satisfeito" a "Completamente satisfeito". No estudo original o índice global desta escala apresentou alfa de Cronbach dentro dos valores estatisticamente indicativos de fiabilidade aceitável. No presente trabalho, o índice global apresenta alfa de Cronbach na amostra comunitária de 0,97 e correlação média interitens (CMII) de 0,46; na amostra clínica, $\alpha=$ 0,99 e $C M I I=0,62$; e, na amostra recolhida online, $\alpha=$ 0,98 e $C M I I=0,50$.

Body Appearance Cognitive Distraction Scale (BACDS; Dove \& Wiederman, 2000). Utilizou-se uma subescala de autorrelato da Cognitive Distraction Scale (QDC) para o estudo da validade discriminante. A escala avalia a distração cognitiva ou interferência durante a atividade sexual e possui 20 itens com uma escala de Likert 6 pontos (entre 1 - "Sempre" e 6 - "Nunca"), que indica quão frequentemente o respondente concorda com a afirmação. Os itens cobrem dois possíveis focos de atenção a que correspondem 10 itens cada: a performance sexual e a aparência corporal, sendo que é este último que se detém a presente investigação. A escolha do instrumento foi orientada pelas diretrizes para a validação de instrumentos referida por Moreira (2004), que aponta a necessidade de utilização de um instrumento que meça um construto relacionado com aquele em estudo. É previsto que as correlações apresentadas entre os dois instrumentos sejam baixas por forma a indicar a existência, quer de relação, quer de discriminação, suficiente entre ambos os construtos. No estudo de Meana e Nunnink (2006), com uma amostra de homens e mulheres, o coeficiente de consistência interna da subescala de Aparência Corporal foi de 0,94. No presente trabalho, os valores encontrados de fiabilidade para a amostra normativa foram de $\alpha=0,84$ e $C M I I=0,44$; na amostra clínica de $\alpha=0,91$ e $C M I I=0,52$; e, na amostra online de $\alpha=0,92$ e $C M I I=0,56$.

\section{Procedimentos}

A versão original do GMREL foi submetida aos procedimentos usuais de tradução e retroversão em quatro fases: (a) tradução independente da versão original por dois especialistas na área e (b) posterior elaboração de uma versão final; (c) retroversão para a língua inglesa por um psicólogo bilingue; e (d) acertos finais. Realizaram-se ainda duas aplicações-piloto, com 15 e 20 estudantes universitários respetivamente, para confirmar se permitia uma compreensão da linguagem adequada.
No Subestudo 1, relativamente à amostra presencial comunitária, foram efetuadas aplicações coletivas $(N=57)$ em instituições de ensino universitário, escolas públicas e uma câmara municipal e aplicações individuais $(N=38)$. Os dados relativos à amostra presencial clínica foram recolhidos em contexto público $(N=27)$ e privado $(N=$ 29). No contexto público, fez-se o pedido de colaboração a uma consulta de sexologia clínica de um Hospital Psiquiátrico da zona de Lisboa, no qual foi apreciado pela Comissão de Ética e do Núcleo de Investigação Científica desta instituição que aprovou o estudo e os procedimentos de recolha de dados. A recolha de dados foi implementada após a consulta de triagem. Em contexto privado, os dados foram recolhidos no consultório de uma terapeuta familiar acreditada pela Sociedade Portuguesa de Terapia Familiar. Em ambos os contextos, público e privado, os clínicos confirmavam a existência de problemas conjugais clinicamente significativos. A todos os participantes das amostras presenciais, era entregue um envelope com o protocolo, o formulário de consentimento, informações sobre o estudo, sobre os procedimentos de preenchimento e devolução.

Foi usada uma plataforma $W e b$ para a recolha de dados do Subestudo 2 (amostra online), previamente testada com cerca de 30 participantes que avaliaram e comentaram a adequabilidade formal e de conteúdo do protocolo. $\mathrm{O}$ estudo foi lançado publicamente após os acertos finais, tendo sido prestada informação acerca dos objetivos e indicado o URL da página do consentimento informado que continha informações sobre intuito, condições de participação no estudo (idade mínima de 18 anos e nacionalidade portuguesa), garantia de não registo de IP, segurança e atualização do servidor. A recolha de dados online prestou-se durante 4 meses. Em ambos os estudos foram assegurados o anonimato e a confidencialidade, foi fornecido o contacto da investigadora e informações sobre serviços de apoio técnico especializado.

\section{Análises Estatísticas}

Considerando os objetivos expostos, são de seguida apresentadas as evidências de validade fatorial, convergente, discriminante, estudo da capacidade discriminativa e estudo da fiabilidade do instrumento. As análises estatísticas foram efetuadas através do uso do SPSS 19.0 (SPSS Inc, Chicago IL, USA), e em ambos os estudos, foram utilizados os procedimentos estatísticos descritos.

Os estudos sobre a estrutura fatorial do GMREL foram realizados através Análise em Componentes Principais $(\mathrm{ACP})$, recorrendo à matriz de correlações de Pearson. Apesar de constituir uma medida global que originalmente é constituída por somente um fator, foi levada a cabo uma análise fatorial exploratória para apurar se todos os itens da GMREL exibem peso fatorial suficiente que justifique a sua inclusão na adaptação portuguesa do instrumento.

Para o estudo da validade discriminante foi analisada a relação entre a GMREL e a BACDS e, para o estudo da validade convergente, foi analisada a relação entre a GMREL e o índice global da EASAVIC. Para a análise da 
Pascoal, P. M., Oliveira, L. B. \& Raposo, C. F. (2015). Evidências de Validade da Global Measure of Relationship Satisfaction (GMREL) em Três Amostras da População Portuguesa.

capacidade discriminativa da GMREL foram empregues diferentes métodos estatísticos. Para a comparação do resultado da GMREL das amostras clínica e comunitária, utilizou-se o teste $T$ de Student para amostras independentes. Efetuou-se ainda uma regressão logística binária a partir do total da GMREL para determinar, por um lado, a probabilidade de detetar se uma pessoa pertence ao grupo clínico e, por outro, a sensibilidade e especificidade do instrumento.

Por fim, foi estudada a Receiver Operating Characteristics Curve (ROC Curve), para verificar a acuidade da GMREL como instrumento de avaliação da satisfação relacional global para diferenciar população clínica da não-clínica. Recorreu-se à análise da ROC Curve devido às dificuldades inerentes ao uso da regressão logística com apenas uma variável preditora, uma vez que esta dificilmente leva a um bom ajustamento do modelo de regressão. No estudo da ROC Curve, para cada resultado nos questionários a curva posiciona a sensibilidade (que deteta os verdadeiros positivos) e a especificidade (que nos dá a taxa de falsos positivos). A área abaixo da curva (area under the curve - AUC) assinala a capacidade de distinguir entre "casos" (com a presença da característica em estudo) e "não casos" (sem a característica em estudo). O índice da AUC pode variar entre 0,5 - que aponta uma possibilidade aleatória de associação, ou seja, nenhum valor diagnóstico, e 1,0 - que indica perfeita discriminação entre "casos" e "não casos". Hosmer e Lemeshow (2000, citados por Maroco, 2007) expõem valores indicativos de discriminação fraca (entre 0,5 e 0,7 ) e discriminação aceitável $(0,7$ a 0,8$)$.

\section{Resultados}

\section{Estudo de Validade}

Validade Fatorial. Na Tabela 2, encontram-se descritos os resultados relativos ao Subestudo 1 de validade fatorial da GMREL através da Análise em Componentes Principais (ACP). De acordo com os resultados expressos, na amostra presencial normativa, foi selecionado um fator, quer pelo critério de Kaiser (valores próprios superiores a um), quer pelo critério da percentagem total de variabilidade explicada adequada (superior a 75\%). Na amostra presencial clínica (ver Tabela 2), também foi selecionado apenas um fator, quer pelo critério de Kaiser (valores próprios superiores a um), quer pelo critério da percentagem total de variabilidade explicada adequada (superior a $75 \%$ ). No Subestudo 2, realizado com a amostra online, os resultados confirmam a estrutura unifatorial da GMREL (ver Tabela 2), quer pelo critério de Kaiser (valores próprios superiores a um), quer pelo critério da percentagem total de variabilidade explicada adequada (superior a 75\%).

Tabela 2

Estrutura Fatorial Final da GMREL na Amostra Presencial Comunitária $(N=95)$, na Amostra Presencial Clínica $(N$ =102) e na Amostra Online $(N=669)$

\begin{tabular}{cccc}
\hline & \multicolumn{2}{c}{ Subestudo 1 } & Subestudo 2 \\
\cline { 2 - 4 } & Amostra Presencial & Amostra Presencial & Clínica \\
Comunitária & Fator 1 & Online \\
\hline Itens & 0,964 & 0,814 & Fator 1 \\
1 & 0,962 & 0,958 & 0,958 \\
2 & 0,954 & 0,960 & 0,956 \\
3 & 0,935 & 0,961 & 0,955 \\
4 & 0,733 & 0,858 & 0,949 \\
5 & 83,56 & 83,24 & 0,837 \\
Percentagem de & & & 86,89 \\
variabilidade explicada & & & \\
\hline
\end{tabular}

Validade Convergente. No Subestudo 1 o resultado da correlação entre a GMREL e o índice total da EASAVIC na amostra presencial comunitária foi de $r=0,79(p<$ $0,001)$ e, na amostra presencial clínica, foi de $r=0,84$ ( $p$ $<0,001$ ), evidenciando que estes instrumentos encontram-se forte e significativamente relacionados. Relativamente ao Subestudo 2, com a amostra recolhida online, o valor do coeficiente de correlação entre a GMREL e o índice global da EASAVIC na amostra online foi de $r=0,78$ ( $p$ $<0,001)$. O valor encontrado, é indicador de uma relação direta, forte e significativa.

Validade Discriminante. No Subestudo 1, o resultado da correlação entre a GMREL e a BACDS na amostra comunitária foi de $r=-0,02(p=0,925)$, um valor esta- 
tisticamente não significativo que indica a inexistência de relação entre as duas medidas. $\mathrm{Na}$ amostra clínica, o valor $r=-0,02(p=0,872)$ revela a inexistência de relação entre as duas variáveis. No Subestudo 2, o resultado da correlação entre a GMREL e a BACDS foi de $r=-0,22$ $(p<0,001)$, um valor indicativo de uma relação inversa, fraca e significativa entre os dois instrumentos.

Capacidade Discriminativa. No Subestudo 1, a média do total da GMREL na amostra comunitária $(M=30,93$; $D P=4,74 ; N=83$ ) é superior à média na amostra clínica $(M=22,61 ; D P=6,66 ; N=56)$. A utilização do teste $T$ de Student revelou que a distribuição dos níveis de satisfação relacional tem valores superiores na amostra comunitária $(t=-7,352$; valor $p \leq 0,001$, magnitude do efeito $=0,669$, $d$ de Cohen $=1,801$ ).

Foi efetuada uma regressão logística binária, tendo como variável dependente a pertença $(N=95)$ ou não pertença $(N=44)$ à amostra clínica. $\mathrm{O}$ modelo de regressão mostrou-se significativo $\left[\chi^{2}(1)=50,458 ; p \leq 0,001\right]$, apresentando uma percentagem de bem classificados de $82,7 \%$, estimada por re-substituição. Este valor é superior à percentagem de bem classificados pela regra da maioria $(65,4 \%)$. A especificidade foi de $79,2 \%$ e a sensibilidade de $65,9 \%$, sendo este último, um valor considerado alto e que parece indicar que este instrumento é sensível para diferenciar a população clínica da não clínica. O teste de Hosmer e Lemeshow revelou resultados significativos $\left[\chi^{2}(7)=12,969 ; p=0,07\right]$. Para que se possa considerar que o modelo é bem ajustado, não se deve rejeitar a hipótese nula do teste de Hosmer e Lemeshow, pelo que, no presente trabalho, os valores encontrados indicam um bom ajustamento do modelo de regressão.

No presente estudo, a ROC Curve apresentou uma área abaixo da curva de 0,86 ( $p<0,001$ e 95\% IC: 0,793$0,933)$, um valor indicador de discriminação forte. $\mathrm{Na}$ Tabela 3 apresenta-se o valor de especificidade e sensibilidade para cada ponto de corte.

Estudo de Fiabilidade. Relativamente ao Subestudo 1 , a avaliação da fiabilidade, que considerou os valores do alfa de Cronbach e de CMII, revelou bons níveis de fiabilidade, quer na amostra comunitária $(N=95) \operatorname{com} \alpha$ $=0,95$ e $C M I I=0,79$, quer na amostra clínica $(N=56)$, $\operatorname{com} \alpha=0,95$ e $C M I I=0,78$. No Subestudo 2, na amostra online $(N=669)$, o alfa de Cronbach apresenta um valor de 0,96 e $C M I I=0,83$.

\section{Discussão}

O presente trabalho procurou escrutar as evidências de validade dos resultados da versão portuguesa da Global Measure of Relationship Satisfaction (GMREL), um instrumento com o propósito da avaliação da satisfação relacional, aqui concebida como a apreciação pessoal e subjetiva da qualidade da relação amorosa (e.g., Lawrance \& Byers, 1995; Narciso \& Ribeiro, 2009). O trabalho foi desenvolvido junto de três amostras distintas da população portuguesa: (a) uma amostra presencial comunitária; (b)
Tabela 3

Pontos de Corte do Instrumento e Resultados de Sensibilidade e Especificidade

\begin{tabular}{|c|c|c|}
\hline Pontos de corte $\geq$ & Sensibilidade & Especificidade \\
\hline 4,0 & 0,000 & 0,000 \\
\hline 5,5 & 0,023 & 0,012 \\
\hline 8,5 & 0,045 & 0,012 \\
\hline 13,0 & 0,068 & 0,012 \\
\hline 16,0 & 0,136 & 0,012 \\
\hline 17,5 & 0,159 & 0,024 \\
\hline 18,5 & 0,182 & 0,024 \\
\hline 19,5 & 0,250 & 0,024 \\
\hline 20,5 & 0,409 & 0,024 \\
\hline 21,5 & 0,432 & 0,036 \\
\hline 22,5 & 0,523 & 0,036 \\
\hline 23,5 & 0,591 & 0,036 \\
\hline 24,5 & 0,614 & 0,036 \\
\hline 25,5 & 0,659 & 0,084 \\
\hline 26,5 & 0,727 & 0,108 \\
\hline 27,5 & 0,750 & 0,169 \\
\hline 28,5 & 0,818 & 0,229 \\
\hline 29,5 & 0,841 & 0,289 \\
\hline 30,5 & 0,864 & 0,458 \\
\hline 31,5 & 0,909 & 0,530 \\
\hline 32,5 & 0,932 & 0,554 \\
\hline 33,5 & 0,955 & 0,627 \\
\hline 34,5 & 0,977 & 0,639 \\
\hline 36,5 & 1,000 & 1,000 \\
\hline
\end{tabular}

uma amostra presencial clínica; e (c) uma amostra comunitária recolhida online.

Iniciou-se o estudo com a análise fatorial do instrumento através da Análise de Componentes Principais (ACP), sendo que a estrutura unifatorial original da GMREL foi replicada nas três amostras em estudo.

Seguidamente recorreu-se ao coeficiente de Pearson com o intuito de examinar a validade de construto do instrumento. Para o efeito, foi analisada a relação da GMREL com o índice global da EASAVIC (Narciso \& Costa, 1996), que avalia a satisfação em áreas da vida conjugal, para a obtenção da validade convergente, e a relação com a subescala Cognitive Distraction Scale da BACDS (Dove \& Wiederman, 2000), que avalia a distração cognitiva ou interferência durante a atividade sexual, para a obtenção da validade discriminante. Relativamente ao primeiro caso, 
Pascoal, P. M., Oliveira, L. B. \& Raposo, C. F. (2015). Evidências de Validade da Global Measure of Relationship Satisfaction (GMREL) em Três Amostras da População Portuguesa.

verificou-se a existência de uma relação direta e forte entre os instrumentos nas três amostras, apesar da relação com a amostra clínica se apresentar mais fraca que as demais relações. A direção destes resultados era esperada, considerando que a GMREL e a EASAVIC partilham semelhantes assunções teóricas concernentes à satisfação relacional, apesar da última ser específica à satisfação conjugal suporta, em termos estatísticos, a validade convergente da GMREL. Já as relações encontradas entre a GMREL e a BACDS foram inversas e fracas, o que sugere que quanto maior o grau de distração negativa, menor a satisfação relacional, apesar da única relação estatisticamente significativa encontrada se reportar à amostra online. Tais resultados suportam a existência de validade discriminante, (i.e., que as duas escalas representam instrumentos distintos, embora relacionados, mas não de forma moderada ou forte), sendo que a relação encontrada na população online é inequívoca nesse sentido.

Para proceder a avaliação da capacidade discriminativa do instrumento no Subestudo 1, foi efetuada a comparação das médias totais do instrumento obtidas nas amostras comunitária e clínica. O teste $T$ de Student demonstrou diferenças significativas entre as duas amostras. $\mathrm{O}$ tamanho do efeito encontrado, de acordo com as orientações de Cohen (1988), é forte, o que indica a alta capacidade do instrumento em diferenciar a amostra clínica da amostra não-clínica e suporta que os resultados deste estudo com a GMREL são genralizáveis a outros contextos (i.e., a probabilidade de existir um erro tipo I é baixa). Foram ainda executadas regressões logísticas binárias com o objetivo de verificar a capacidade preditiva do instrumento (i.e., indicar a que grupo pertence cada participante - clínico/não clínico, nas amostras presenciais; ou amostra online). Em todas as amostras, o resultado da regressão logística binária confirma a adequação do instrumento para a correta distinção dos participantes, apresentando uma especificidade de $79,2 \%$ e sensibilidade $65,9 \%$. Estes resultados parecem indicar que a GMREL revela forte sensibilidade para discriminar entre situações com e sem dificuldade relacional. Os resultados da ROC Curve, suportam estes mesmo são indicativos de que a GMREL tem boa sensibilidade para discriminar a população clínica da não clínica quando os problemas sexuais são o motivo que leva o casal a procurar ajuda e que estes reconhecem haver problemas relacionais. A leitura da tabela para a selecção do melhor ponto de corte deve ter em conta os objectivos para os quais se pretende utilizar a GMREL. No contexto de investigação, em que a GMREL pode ser um instrumento útil para fazer o screening de pessoas com problemas relacionais associados às queixas sexuais, um ponte de corte de 34, por exemplo, apesar de apresentar alta sensibilidade, é indicador de alta especificidade o que implicaria um controle pouco eficaz de falsos positivos. Deve ser o investigador a seleccionar o ponto de corte mais adequado aos seus objectivos e mantendo presente que apesar dos bons resultados ao nível da sensibilidade e especificidade da GMREL, o presente trabalho apresenta limitações que não devem ser negligenciadas. No estudo de fiabilidade a versão portuguesa da GMREL revelou-se uma medida consistente, uma vez que apresentou valores de alfa de Cronbach elevados em todas as amostras, mais especificamente de 0,95 na amostra comunitária presencial e clínica e de 0,96 na amostra online. Os valores de CMII (nas amostras comunitária $=0,79 ;$ clínica $=0,78$; e online $=0,83$ ), que correspondem à média das correlações entre cada par de itens, são elevados, indicando assim homogeneidade entre estes.

O presente estudo apresenta limitações várias. Salientamos em primeiro lugar o facto das amostras estudadas serem de conveniência e não serem representativas da população portuguesa. Ainda o facto de a população clínica apresentar dificuldades sexuais como queixa principal, podendo apenas indicar que os valores da validade discriminativa do instrumento encontrados se reportam à população com problemas relacionais associados a dificuldades sexuais. Futuramente as amostras recolhidas deverão incluir maior dimensão e diversidade, como ao nível dos casais poliamorosos ou com elementos do mesmo sexo, considerando que um dos limites da presente investigação prende-se com a recolha de dados ter sido realizada no âmbito de uma consulta de sexologia clínica. Seria igualmente pertinente verificar se há especificidades na satisfação relacional em diferentes quadros clínicos, nomeadamente ao nível da comunicação ou da gestão da parentalidade. Desta forma poder-se-á discernir se os resultados obtidos são reproduzidos em indivíduos com outras problemáticas relacionais.

Em suma, considera-se que o instrumento pode ser utilizado em contexto de investigação específica com amostras clínicas, pois permite com clareza diferenciar pessoas com e sem problemas relacionais. A pertinência dos estudos das evidências de validade para a população portuguesa da GMREL, remete grandemente para possibilidade de aplicação nos contextos clínico e de investigação, considerando, nomeadamente, que os instrumentos de satisfação relacional validados em língua portuguesa são escassos e longos, contrariamente à escala em questão, que com apenas 5 itens permite avaliar fielmente a satisfação relacional. Além disso, o facto de a GMREL poder ser empregue em ambos sexos e em diferentes estruturas relacionais, a sua fácil compreensão e rapidez de resposta, e os resultados fornecidos pelos estudos de validade, indicam a adequação do instrumento ao universo português.

\section{Referências}

Bradbury, T. N., Fincham, F. D., \& Beach, S. R. (2000). Research on the nature and determinants of marital satisfaction: A decade in review. Journal of Marriage and the Family, 62(4), 964-980. doi:10.1111/j.1741-3737.2000.00964.x

Byers, E. S. (2005). Relationship satisfaction and sexual satisfaction: A Longitudinal Study of Individuals in Long-Term Relationships. Journal of Sex Research, 42(2), 113-118. doi:10.1080/00224490509552264 
Byers, E. S., \& Demmons, S. (1999). Sexual satisfaction and sexual self-disclosure within dating relationships. Journal of Sex Research, 36(2), 180-189. doi:10.1080/00224499909551983

Byers, E. S., Demmons, S., \& Lawrance, K. A. (1998). Sexual satisfaction within dating relationships: A Test of the Interpersonal Exchange Model of Sexual Satisfaction. Journal of Social and Personal Relationships, 15(2), 257-267. doi:10.1177/0265407598152008

Byers, E. S., \& MacNeil, S. (2006). Further validation of the Interpersonal Exchange Model of Sexual Satisfaction. Journal of Sex \& Marital Therapy, 32(1), 53-69. doi:10.1080/00926230500232917

Christensen, H., Griffiths, K. M., \& Jorm, A. F. (2004). Delivering interventions for depression by using the internet: Randomised controlled trial. British Medical Journal, 328, 265-269. doi:10.1136/bmj.37945.566632.EE

Cohen, J. (1988). Statistical power analysis for the behavioral sciences ( $2^{\text {nd }}$ ed.). Hillsdale, NJ: Lawrence Earlbaum Associates.

Cusack, C. E., Hughes, J. L., \& Cook, R. E. (2012). Components of love and relationship satisfaction: Lesbians and heterosexual women. Psi Chi, the International Honor Society in Psychology, 17(4), 171-179. Retrieved from http://connection.ebscohost.com/c/articles/85395308/components-loverelationship-satisfaction-lesbians-heterosexual-women

Dove, N. L., \& Wiederman, M. W. (2000). Cognitive distraction and women's sexual functioning. Journal of Sex \& Marital Therapy, 26(1), 67-78. doi:10.1080/009262300278650

Fincham, F. D., \& Beach, S. R. (2010). Marriage in the new millennium: A decade in review. Journal of Marriage \& Family, 72(3), 630-649. doi:10.1111/j.1741-3737.2010.00722.x

Funk, J. L., \& Rogge, R. D. (2007). Testing the ruler with item response theory: Increasing precision of measurement for relationship satisfaction with the Couples Satisfaction Index. Journal of Family Psychology, 21(4), 572-583. doi:10.1037/0893-3200.21.4.572

Gomez, R., \& Leal, I. (2008). Ajustamento conjugal: Características psicométricas da versão portuguesa da Dyadic Adjustment Scale. Análise Psicológica, 4(26), 625-638. Recuperado em http://www.scielo.oces.mctes.pt/pdf/aps/v26n4/v26n4a08.pdf

Hendrick, S. S. (1988). A generic measure of relationship satisfaction. Journal of Marriage and Family, 50(1), 93-98. doi: $10.2307 / 352430$

Hudson, W. W., Harrison, D. F., \& Crosscup, P. C. (1981). A short-form Scale to Measure Sexual Discord in Dyadic Relationships. Journal of Sex Research, 17(2), 157-174. doi:10.1080/00224498109551110

Laub, J. H., Nagin, D. S., \& Sampson, R. J. (1998). Trajectories of change in criminal offending: Good marriages and the desistance process. American Sociological Review 63(2), 225-238. Retrieved from http://www.jstor.org/discover/10. $2307 / 2657324$ ?uid $=3738880$ \&uid $=2132$ \&uid $=2 \&$ uid $=70 \&$ uid $=4 \&$ sid $=21104055122461$

Lawrance, K. A., \& Byers, E. S. (1995). Sexual satisfaction in long-term heterosexual relationships: The interpersonal exchange model of sexual satisfaction. Personal Relationships, 2(4), 267-285. doi:10.1111/j.1475-6811.1995.tb00092.x

MacNeil, S., \& Byers, E. S. (2009). Role of sexual selfdisclosure in the sexual satisfaction of long-term heterosexual couples. Journal of Sex Research, 46(1), 3-14. doi:10.1080/00224490802398399

Maroco, J. (2007). Análise estatística com utilização do SPSS. Lisboa, Portugal: Sílabo.
McGuire, J., \& Barber, B. (2010). A person-centered approach to the multifaceted nature of young adult sexual behavior. Journal of Sex Research, 47(4), 301-313. doi:10.1080/00224490903062266

Meana, M., \& Nunnink, S. E. (2006). Gender differences in the content of cognitive distraction during sex. Journal of Sex Research, 43(1), 59-67. doi:10.1080/00224490609552299

Moreira, J. (2004). Questionários: Teoria e prática. Coimbra, Portugal: Almedina.

Narciso, I., \& Costa, M. E. (1996). Amores satisfeitos, mas não perfeitos. Cadernos de Consulta Psicológica, 12, $115-$ 130. Recuperado de http://repositorio-aberto.up.pt/bitstream/10216/15550/2/31582.pdf

Narciso, I., \& Ribeiro, M. T. (2009). Olhares sobre a conjugalidade. Lisboa, Portugal: Coisas de Ler.

Peck, S. R., Shaffer, D. R., \& Williamson, G. M. (2004). Sexual satisfaction and relationship satisfaction in dating couples: The contributions of relationship communality and favorability of sexual exchanges. Journal of Psychology \& Human Sexuality, 16(4), 17-37. doi:10.1300/J056v16n04_02

Renaud, C., Byers, E. S., \& Pan, S. (1997). Sexual and relationship satisfaction in Mainland China. Journal of Sex Research, 34(4), 399-410. doi:10.1080/00224499709551907

Spanier, G. B. (1976). Measuring dyadic adjustment: New scales for assessing the quality of marriage and similar dyads. Journal of Marriage and the Family, 38(1), 15-28. doi:10.2307/35054

Vaughn, M. J., \& Baier, M. E. (1999). Reliability and validity of the relationship assessment scale. American Journal of Family Therapy, 27(1), 137-147. doi:10.1080/019261899262023

Wade, T. J., \& Pevalin, D. J. (2004). Marital transitions and mental health. Journal of Health and Social Behavior, 45, 155-170. doi:10.1177/002214650404500203

Whisman, M. A. (2007). Marital distress and DSM-IV psychiatric disorders in a population-based national survey. Journal of Abnormal Psychology, 116, 638-643. doi:10.1037/0021843X.116.3.638

World Health Organization. (2006). Defining sexual health: Report of a technical consultation on sexual health, 28-31 January 2002. Retrieved from http://whqlibdoc.who.int/ hq/2010/who_rhr_10.12_eng.pdf 